\title{
Du Volksmärchen à la pop culture. Reprises et hybridations dans l'œuvre de Yann Legendre, Grimm, Contes choisis
}

\section{Anne-Sophie Gomez}

\section{(2) OpenEdition}

Journals

Édition électronique

URL : https://journals.openedition.org/edl/1590

DOI : $10.4000 /$ edl. 1590

ISSN : 2296-5084

Éditeur

Université de Lausanne

\section{Édition imprimée}

Date de publication : 15 septembre 2019

Pagination : 81-98

ISBN : 978-2-940331-71-0

ISSN : 0014-2026

Référence électronique

Anne-Sophie Gomez, « Du Volksmärchen à la pop culture. Reprises et hybridations dans l'œuvre de Yann Legendre, Grimm, Contes choisis », Études de lettres [En ligne], 310 | 2019, mis en ligne le 15 septembre 2021, consulté le 22 septembre 2021. URL : http://journals.openedition.org/edl/1590 DOI : https://doi.org/10.4000/edl.1590

Ce document a été généré automatiquement le 22 septembre 2021.

(c) Études de lettres 


\title{
Du Volksmärchen à la pop culture. Reprises et hybridations dans l'œuvre de Yann Legendre, Grimm, Contes choisis
}

\author{
Anne-Sophie Gomez
}

1 Populaires, les contes des frères Grimm le sont assurément, moins par leur origine, dont l'authenticité orale a été largement remise en cause par les chercheurs dans le sillage des travaux de Heinz Rölleke ${ }^{1}$, que par leur réception et leur diffusion progressive au sein de l'imaginaire collectif. Inscrits au registre de la Mémoire du monde par l'UNESCO en $2005^{2}$, les Kinder- und Hausmärchen ont largement dépassé, et ce dès le XIX ${ }^{e}$ siècle, les frontières de l'aire germanophone, grâce à des traductions en plus de 160 langues et dialectes culturels. Afin de justifier sa décision, l'UNESCO précise que ces contes «sont, à côté de la Bible de Luther, le livre de culture allemande le plus connu et le plus répandu dans le monde $»^{3}$. En 2011, à la veille du bicentenaire de la première parution des contes, les éditions d'art Taschen ont publié une belle anthologie illustrée ${ }^{4}$ qui est sortie simultanément en Allemagne, en Espagne, en France, en Italie et au Royaume Uni «pour viser le public international concerné par une œuvre promue au rang d'objet patrimonial universel $»^{5}$. En nous penchant sur le recueil illustré Grimm, Contes choisis de Yann Legendre, paru en 2014 aux éditions Textuel, nous souhaitons montrer que la démarche de l'illustrateur français rejoint à bien des égards le projet initial des Frères Grimm et le tour que ce projet a pris au fil des éditions successives des Kinder- und Hausmärchen.

2 Yann Legendre est un illustrateur français né en 1972. Son travail lui a rapidement valu une reconnaissance internationale; publié dans le Wall Street Journal, le New Yorker et le New York Times, il est aussi l'auteur de nombreuses affiches pour le théâtre et le cinéma, d'illustrations pour des couvertures de livres ou des pochettes de disques $^{6}$, et son œuvre a déjà fait l'objet de plusieurs expositions à travers le monde. Yann Legendre travaille exclusivement sur tablette graphique, donc sur un support numérique, sans 
recours au papier ni à un croquis préparatoire. Dans le recueil Grimm, Contes choisis, composé d'une sélection de vingt contes, il propose des illustrations en pleine page, entre une et cinq par conte, en fonction de la longueur du texte, voire sur une double page, ainsi que des médaillons et des ornements. Ces derniers n'ont pas été réalisés par Legendre lui-même mais ils sont le fruit d'une collaboration avec l'artiste Lance Rutter. Précisons ici que les contes illustrés par Yann Legendre ont été publiés en 2014 à la fois en français - dans la traduction de Natacha Rimasson-Fertin ${ }^{7}$, avec l'autorisation des éditions José Corti - et en anglais chez Rockport Publishers.

Cette contribution se propose d'interroger en deux temps ce qui, dans l'œuvre de Yann Legendre, relève de la reprise voire de la reproduction, et ce qui en fait l'originalité, l'actualité et l'accessibilité à un public varié, du fait de la combinaison des différents horizons de référence que l'illustrateur convoque. Car, comme le dit Peggy Davis, citée par Anne Defrance dans son introduction au numéro de la revue Féeries consacrée à l'illustration du conte merveilleux français: «[...] l'étude du rôle et des enjeux de l'illustration comme pratique sociale, culturelle voire intermédiale, s'inscrit dans un paradigme d'histoire sociale de l'art et de la culture visuelle $»^{8}$.

\section{L'éclectisme des références intericoniques de Yann Legendre}

4 L'intericonicité désigne la mise en perspective d'une image au sein d'un réseau d'images qui la précèdent, sur le modèle de l'intertextualité littéraire. Mathilde Arrivé, auteur d'un article fort éclairant sur le sujet, définit en ces termes le concept :

[...] l'intericonicité n'est pas une caractéristique intrinsèque [...] mais le résultat de déplacements graphiques non-linéaires dans l'espace, dans le temps et dans les formes, exigeant de s'interroger sur la mobilité des images [...], leur historicité, leur plasticité, leur dynamisme et leur productivité, le tout dans une histoire des images conçue comme réseau plutôt que comme cycle ou ligne droite 9 .

5 Spécialiste de l'illustration anglaise des contes des Frères Grimm au XIX ${ }^{e}$ siècle, François Fièvre écrit pour sa part à propos du travail d'illustration des Grimm par Maurice Sendak :

[...] ces œuvres où l'artiste est moins libre de "faire ce qu'il veut» sont intéressantes [...] parce qu'elles montrent l'illustrateur aux prises avec un imaginaire différent du sien ${ }^{10}$.

Il s'agit en effet, dans le cas d'un illustrateur extérieur :

[...] d'accorder sa fantaisie propre à l'univers textuel d'un autre, accord qui demande tact, savoir-faire et sens de l'équilibre. Sens de l'écoute également, aussi bien vis-à-vis du texte des Grimm que de la tradition artistique et littéraire dont celui-ci procède et qu'il précède $[. . .]^{11}$.

6 Parmi les horizons de référence de Yann Legendre, on distingue aisément de multiples emprunts à la pop culture, qui vont de la citation presque littérale à la recomposition, pour reprendre deux des catégories de la typologie établie par François Fièvre. De tels emprunts ou transferts graphiques sont au service d'un dialogue entre plusieurs époques et plusieurs esthétiques, qui entrent ainsi en résonance. Pour commencer, Yann Legendre ne cache pas son admiration pour les auteurs de comics des années 1960. Il déclare par exemple, rendant hommage à Jack Kirby :

Kirby's work is so bold, almost like a nuclear attack exploding in your face. My style is a combination of that old-fashioned line work with a more contemporary tool ${ }^{12}$. 
7 En ce qui concerne son recueil de contes, nous avons souhaité mettre l'accent sur la covergirl placée au centre de la couverture du volume. Il s'agit de la princesse du « Roi Grenouille », représentée non comme la fillette du texte, jouant au bord du puits ${ }^{13}$ mais, sur le mode de la citation intericonique explicite, comme une jeune femme déjà parée de bien des atouts de la féminité et dont les traits ne sont pas sans rappeler ceux de l'amazone Diana Prince, alias "Wonder Woman ", l'héroïne de DC comics, créée dès 1941 par William Moulton Marston, et qui s'est progressivement imposée comme "l'héroöne la plus reconnaissable de l'Amérique » ${ }^{14}$ (fig. 1).

$<$ Image en attente de droits>

Fig. 1 - « Le Roi Grenouille ». ( ) Yann Legendre, illustrations reproduites avec l'aimable autorisation de l'auteur.

8 Parmi les similitudes les plus évidentes figurent la longue chevelure, le diadème, les épaules dénudées, les sourcils fins, dessinés et levés, les yeux écarquillés et la bouche entrouverte. Outre leur érotisme assumé, les covergirls se caractérisent par la situation périlleuse dans laquelle elles semblent plongées dès la couverture, situation qui ne se résoudra que par l'achat du numéro concerné. Chez Yann Legendre, il s'agit toutefois peut-être moins de l'imminence d'un péril à combattre que de surprise ou, déjà, d'un dégoût pour le batracien placé au premier plan et doué de parole. Interrogé par e-mail sur cette possible intericonicité, l'illustrateur nous a confié avoir avant tout pensé au visage épouvanté de l'actrice Susan Sarandon, héroïne en 1975 de la comédie musicale The Rocky Horror Picture Show, dans laquelle elle incarnait le personnage de Janet Weiss.

9 Autre source d'inspiration issue de la pop culture, le cinéma d'animation des studios Disney, dont on identifie à la fois des citations et des recompositions. À la Blanche Neige de Walt Disney, Yann Legendre emprunte la posture et la couleur du corsage de l'héroïne, même si la pomme, tombée au sol chez Disney, a subi un changement d'échelle et apparaît désormais comme un cercueil chez Legendre ${ }^{15}$. Il s'agit donc dans ce cas d'une recomposition. Dans ses illustrations de "Cendrillon", Yann Legendre reprend la coiffure, la blondeur de l'héroïne telle que la montre la version des studios Disney, ainsi que la robe de la jeune fille dans l'illustration en pleine page de la scène de danse avec le prince ${ }^{16}$. On notera d'autre part, sur l'illustration où Cendrillon se tient pieds nus dans sa cuisine, la présence d'une citrouille posée sur le sol. Cet élément est certes absent du texte des Grimm, mais il figure chez Perrault et Disney. Dans le conte de Perrault en 1697, la citrouille évidée fait en effet office de moyen de transport, moyennant un petit coup de baguette magique ${ }^{17}$. Chez les Grimm en revanche c'est, dans la version de 1812, un attelage de chevaux qui conduit Cendrillon au bal. Comment dès lors expliquer l'hybridation plus ou moins consciente de la version française et de la version allemande du conte? Cela tient peut-être au fait que plusieurs artistes majeurs comme Gustave Doré ${ }^{18}$ et Arthur Rackham ${ }^{19}$ ont représenté l'épisode de la citrouille que l'on creuse ou qui est sur le point de se transformer en carrosse, exerçant probablement une forte influence visuelle sur des générations plus jeunes et éventuellement moins familières des textes-source, au point de provoquer une confusion et une contamination. Il en est de même pour la souris représentée par Yann Legendre en bas dans le cadre de l'image et qui rappelle la métamorphose de ces petits

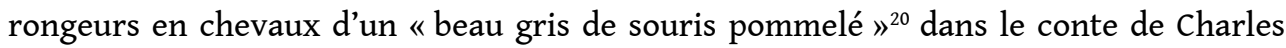
Perrault.

10 Dans l'illustration de "Blanche-Neige " par Yann Legendre, on semble d'autre part déceler l'influence du pop-artiste Claes Oldenburg, qui a principalement travaillé sur 
des objets humbles et quotidiens, ainsi que sur des aliments populaires, comme le hamburger, les frites au ketchup ou les glaces. L'une des caractéristiques de son œuvre est sa prédilection pour le gigantisme. Mais les dimensions de ses œuvres n'empêchent pas un certain réalisme, sous-tendu par la volonté de s'opposer aux sculptures abstraites, très prisées à l'époque. Parmi ces œuvres monumentales exposées uniquement dans des lieux publics, on peut citer la pince à linge, le bâton de rouge à lèvres ou, ce qui nous intéressera plus directement par rapport aux contes, la pomme. Agrandir l'objet, c'est, pour Claes Oldenburg, lui conférer une dynamique, comme dans le cas de l'œuvre Apple Core (1992), co-signée avec Coosje van Bruggen, et qui consiste en un trognon de pomme géant, exposé de manière permanente devant le musée d'Israël à Jérusalem. On pourrait aussi souligner qu'un autre représentant du pop art, précisément nommé Billy Apple du fait de la place centrale qu'il ménage à ce fruit dans son œuvre, a pu inspirer Legendre au moment de composer son illustration pour «Blanche-Neige ». Dans la série en trois temps Two Minutes 33 Seconds (Red), réalisée entre 1962 et 2010, l'artiste Néo-Zélandais montre en effet les étapes de la consommation d'une pomme rouge, sorte de vanité contemporaine qui pourrait faire écho au rôle funeste joué par le fruit dans le conte des frères Grimm. Il faut dire que ce choix de la pomme plutôt que du cercueil rend moins définitive et plus ambiguë l'interprétation du conte par le lecteur: Blanche-Neige, lointaine cousine du Dormeur du val, dort-elle? Autre intertextualité rimbaldienne venant ici s'ajouter à l'intericonicité pop, le tapis de mousse de la forêt paraît si lisse et bleu que la Blanche-Neige de Legendre semble y flotter telle une Ophélia à la dérive (fig. 2).

$<$ Image en attente de droits>

Fig. 2 - «Blanche-Neige ». () Yann Legendre, illustrations reproduites avec l'aimable autorisation de l'auteur.

Mais à l'heure où Yann Legendre a créé ses illustrations des contes, un autre medium constituait, depuis plusieurs années déjà, une voie de perméabilité entre la pop culture et les textes des Grimm. Il s'agit d'Internet et du phénomène de " fluidité numérique " $^{21}$ qu'engendre ce dernier, permettant, à l'heure du digital turn, des « visibilités toujours plus décloisonnées ", selon les termes de Mathilde Arrivé22. En effet, le chat du conte "Chat et souris associés " ${ }^{23}$ n'est pas sans rappeler la moue si caractéristique du grumpy cat, ce « chat grincheux » que son expression faciale a rendu célèbre depuis 2012 grâce aux photos postées par son propriétaire ${ }^{24}$ (fig. 3).

<Image en attente de droits>

Fig. 3 - «Chat et souris associés ». (C) Yann Legendre, illustrations reproduites avec l'aimable autorisation de l'auteur.

12 Un tel anachronisme - 200 ans d'écart entre la première parution des contes et l'apparition sur la toile de ce félin-star (malgré lui) - est fécond précisément par le décalage, l'effet de surprise burlesque et l'inattendu qu'il provoque et qui semble aller à l'encontre de la prophétie selon laquelle à « l'ère des [...] mèmes propagés en ligne par la reprise et la répétition [...] l'abondance (quantitative) [...] des images serait le signe le plus flagrant de leur appauvrissement (qualitatif) ${ }^{25}$. Car «en étant ainsi recontextualisée, remotivée et resémantisée, l'image change de régime de discours, de cahier des charges et de public, et elle s'ouvre à d'autres modes de socialisation tout en se rendant disponible à de nouveaux usages ${ }^{26}$.

13 Si l'on regarde maintenant "Le Petit Chaperon rouge ", on constate que la capuche de l'hérö̈ne évoque davantage une adolescente qu'une fillette ${ }^{27}$, non sans faire écho aux 
codes du streetwear, ce style vestimentaire urbain apparu à New York dans les années 1980 (fig. 4).

$<$ Image en attente de droits>

Fig. 4 - « Le petit chaperon rouge ». () Yann Legendre, illustrations reproduites avec l'aimable autorisation de l'auteur.

Dans un entretien, l'illustrateur explique d'ailleurs qu'il n'a pas voulu faire de l'héroöne une fillette fragile mais plutôt une jeune femme déterminée, comme on pourrait en voir en train d'arpenter les rues de New York:

"In the story, there is nothing saying that the girl is fragile or afraid of the wolf, so I didn't want to illustrate her as a delicate little flower", he says. Legendre drew her as a determined young woman in a hoodie - a character who appears to have just walked off the streets of New York, ready to face the evil hiding beneath her grandmother's bedsheets ${ }^{28}$.

15 Et dans le conte du "Vaillant petit tailleur ", l'image en pleine page représentant le sanglier ${ }^{29}$ rappelle, du fait du cadrage en gros plan de la tête, mais aussi à cause des défenses et du groin proéminents, le genre du trophée de chasse fixé au mur, si populaire dans les pays germaniques, accréditant l'hypothèse selon laquelle, d'après Mathilde Arrivé, il existerait :

[...] des zones de porosité et de friction des codes graphiques et culturels, des «infiltrations mutuelles» entre images, de leurs voisinages parfois erratiques, débordant largement les frontières de genres, d'écoles, de mouvements et de périodes, et même de médiums $\mathrm{s}^{30}$.

16 Or à ces références identifiables par un vaste public s'ajoutent, et c'est ce qui fait toute la complexité et la richesse des illustrations de Yann Legendre, des références plus classiques et érudites, qui témoignent d'une bonne connaissance de l'histoire de l'art européen. Nous prendrons ici l'exemple de Gustave Doré et de ses gravures et tableaux illustrant le «Chaperon rouge » de Perrault ${ }^{31}$. On distingue en effet clairement dans la proposition de Legendre des emprunts dans le traitement de la coiffe et du lorgnon de la grand-mère, ainsi que dans le geste de l'héroïne, dont la main touche les draps.

\section{Une hybridation stylistique}

17 Le recueil illustré par Yann Legendre propose donc un télescopage parfaitement assumé de l'Europe et de l'Amérique, laquelle représente une de ses principales sources d'inspiration consciente :

If you're looking to learn about art between the 14th and 18th century, you go to France, but if you're looking to learn about contemporary illustration, you go to the States [...] In the 1950s and 1960s, everything exploded in the U.S., and I still find inspiration in book covers by Chip Kidd, in graphic novels, and American artwork all of $i^{32}$.

18 Yann Legendre assume aussi dans son œuvre le télescopage entre différentes époques, par exemple entre les gravures de Doré, les ornements hérités du Jugendstil, et l'art contemporain. Même si Legendre n'a pas lui-même réalisé les ornements de son recueil, c'est lui qui a eu l'idée de leur insertion et de leur fonction, qui n'est pas seulement décorative, mais aussi narrative. Yann Legendre a en effet opté pour des plans resserrés avec une relative absence de profondeur. Il a aussi fait le choix de la stylisation - la forêt dans "Blanche-Neige » est ainsi suggérée grâce à la verticalité de seulement huit troncs d'arbre ${ }^{33}$. 
19 Aussi les éléments dessinés dans les cadres des illustrations en pleine page et dans les ornements ont-ils en partie pour fonction d'apporter quelques clés de contexte ou de narration: c'est le cas de la balle d'or dans le "Roi Grenouille», ou du peigne dans "L'Ondine de l'étang", qui surmontent tous les deux une illustration en pleine page ${ }^{34}$. Outre la part importante prise par les végétaux dans les ornements, on est aussi frappé par la chevelure de certaines héroïnes de Legendre, qui n'est pas sans rappeler le traitement réservé par Alphonse Mucha aux cheveux féminins. Et cela, qu'il s'agisse de la chevelure pour ainsi dire ramifiée de la princesse en couverture du recueil ou bien de la coiffure travaillée de la "Jeune fille sans mains », image sur laquelle la circularité du halo lumineux fait écho à un motif également prisé chez l'affichiste tchèque ${ }^{35}$. Interrogé sur la richesse de ses illustrations, Yann Legendre répond que celle-ci nait justement de la place ménagée à l'imaginaire par la concision et l'oralité - ou du moins la vocation orale - du texte original :

The Grimm stories were designed to be told, not read. [...] The way they're written is the way we talk, and because there's so little detail, I can create the detail and interpret the story through the illustration ${ }^{36}$.

L'intericonicité apparaît ainsi comme un dialogue fructueux, permettant de faire entrer en résonance différents horizons artistiques ainsi que différentes époques et aires géographiques, même si ces dernières peuvent à juste titre paraitre éloignées de la culture-source des textes illustrés.

21 Tout comme l'avaient fait les frères Grimm dans leurs Kinder- und Hausmärchen, Yann Legendre a sélectionné, pour son recueil, aussi bien des contes de fées que des récits plus triviaux. Ce faisant, il a souhaité, par son geste anthologique, ne pas réduire les contes aux textes canoniques, mais donner à voir et à entendre des textes moins connus, comme par exemple "La Rave » ou encore le "Conte de la petite souris, du petit oiseau et de la saucisse ». Dans ce dernier, à la différence de Walter Crane par exemple, Yann Legendre ne procède pas à une anthropomorphisation directe du corps de la saucisse, mais il la représente assise dans un fauteuil dont le style bourgeois contribue à accentuer le décalage burlesque entre la nature du personnage et sa position sociale plutôt confortable. Le texte dit en effet: «Il était une fois une petite souris, un petit oiseau et une saucisse qui s'étaient retrouvés ensemble. Ils s'étaient mis en ménage, avaient mené pendant longtemps une vie paisible et délicieuse, et amassé des biens $\aleph^{37}$. Sans compter, comble de l'ironie, que c'est à la saucisse qu'incombe la tâche de préparer le repas, tâche qu'elle accomplit avec zèle. Mais Yann Legendre a aussi retenu d'autres contes à l'étrangeté parfois troublante, comme "L'Ondine de l'étang ", dont la chevelure évoque à nouveau les femmes représentées par Alfons Mucha.

Il faut ici rappeler que les frères Grimm avaient souhaité, avec leur Kinder- und Hausmärchen, aller à l'encontre de la préciosité d'un Perrault par exemple. Cela passait notamment par le fait d'assumer la langue vernaculaire et le dialecte. Car les Grimm, ne cachant pas l'ambition ethnographique de leur projet de sauvegarde de la tradition orale, percevaient les contes comme l'émanation d'un Volksgeist (littéralement, «l'esprit du peuple »). Il semble donc, à travers les différents exemples que nous avons mis en avant, que Yann Legendre cherche à émuler ce projet. En effet, si l'illustrateur a injecté des éléments populaires dans ses propositions graphiques, il ne renie pas non plus un certain héritage culturel dans lequel il a pu baigner du fait de son milieu d'origine et de sa formation : 
Most of my education in art and illustration comes from my parents. My father was an architect who also taught at an art school. Artists and famous creative people would visit for weeks at a time to work on paintings or sculptures, so I was surrounded by creativity ${ }^{38}$.

Le volume de Yann Legendre puise donc dans une inspiration éclectique, qui est à la fois populaire et érudite, à l'image des Kinder- und Hausmärchen des frères Grimm. De même que l'on retrouve chez les Grimm l'influence de Perrault et des farces populaires, il réussit, par ses emprunts et ses recompositions, la synthèse de Gustave Doré et de la culture populaire. En effet, à ses yeux, il n'existe pas de cloisonnement entre l'art des musées et celui qui est apprécié par le grand-public. Ses lecteurs-cibles ne sont pas nécessairement des connaisseurs de l'histoire de l'art, et l'on peut lire et apprécier les contes à différents niveaux, que l'on soit ou non familier avec le contexte socio-culturel de l'époque de leur écriture, que l'on connaisse ou non leur réseau intertextuel ou les contes-types répertoriés par les folkloristes. Le volume de Yann Legendre est avant tout un beau livre, un objet travaillé qui reste toutefois accessible grâce à l'éclectisme de ses références. L'un des objectifs majeurs du pop art était d'abolir la distance entre la vie et l'art, sans exclure du champ de ce dernier la culture de masse. Yann Legendre semble pleinement souscrire à cet objectif en nous livrant sa vision des contes, qu'il ne s'agit plus de renouveler mais plutôt de recomposer, à partir de sources multiples qu'il est possible de combiner afin de faire naître, chez les spectateurs de ses illustrations, ce que l'on pourrait appeler une "conversation ${ }^{39}$ doublée d'une étrange familiarité, entre reproduction et singularité. Pour Yann Legendre, il n'existe pas, en effet, de hiatus ni de cloisonnement entre la culture savante - celle documentée par l'histoire de l'art et les musées - et une culture plus vernaculaire, voire triviale, mais qui imprègne, au même titre que l'art académique, la société et l'imaginaire créatif.

\section{BIBLIOGRAPHIE}

\section{Sources}

GRIMM, Jacob et Wilhelm, Contes pour les enfants et la maison, trad. Natacha Rimasson-Fertin, 2 vols, Paris, Corti, 2009.

LEGENDRE, Yann, Grimm, Contes choisis, Paris, Éditions Textuel, 2014 (trad. anglaise : Grimm's Fairy Tales, Gloucester, Rockport Publishers, 2014 [2017 pour la version au format poche]).

-, Entretien avec Scott Kirkwood (« Yann Legendre's Modern-Day Woodcuts »), 2016 (<http:// create.adobe.com/2016/10/25/yann_legendre_s_modern_day_woodcut_style.html>).

PERRAULT, Charles, «Cendrillon », in Histoires, ou Contes du tems passé : avec des moralités, La Haye, 1742. 


\section{Travaux}

ARRIVÉ, Mathilde, «L'intelligence des images - l'intericonicité, enjeux et méthodes », E-rea [En ligne], 13.1.2015, mis en ligne le 15 décembre 2015 (<http://journals.openedition.org/erea/ $4620>$ ).

CONNAN-PINTADO Christiane, TAUVERON Catherine, Fortune des Contes des Grimm en France. Formes et enjeux des rééditions, reformulations, réécritures dans la littérature de jeunesse, ClermontFerrand, Presses universitaires Blaise Pascal, 2013.

DEFRANCE, Anne, « Introduction : l'illustration du conte merveilleux français des XVII ${ }^{\mathrm{e}}$ et XVIII siècles : un objet de recherche en pleine expansion », Féeries, 11 (2014), p. 9-39.

FIÈVRE, François, «L'image dans l'image : l'intericonicité dans l'illustration des contes de Grimm par Maurice Sendak », in Vies et métamorphoses des Contes de Grimm. Traductions, réception, adaptations, éd. par Dominique Peyrache-Leborgne, Rennes, Presses universitaires de Rennes, 2017, p. 145-167.

GUNTHERT, André, « L'image conversationnelle. Les nouveaux usages de la photographie numérique », Études photographiques, 31 (2014), p. 55-71.

RÖLLEKE, Heinz, Die Märchen der Brüder Grimm, München/Zürich, Artemis Verlag, 1985. SIMONSON, Louise, Covergirls : les héroïnes de DC Comics, Paris, Urban Comics, 2015.

\section{NOTES}

1. H. Rölleke, Die Märchen der Brüder Grimm.

2. < http://www.unesco.org/new/fr/member-states/single-view/news/ twenty_nine_new_documentary_collections_inscribed_on_the_mem/> (consulté le 11 février 2019).

3. < http://www.unesco.org/new/fr/communication-and-information/memory-of-the-world/ register/full-list-of-registered-heritage/registered-heritage-page-4/kinder-und-hausmaerchenchildrens-and-household-tales/> (consulté le 11 février 2019).

4. $\quad$ https://www.taschen.com/pages/fr/catalogue/children/all/48611/ facts.les_contes_des_freres_grimm.htm> (consulté le 11 février 2019).

5. Ch. Connan-Pintado, C. Tauveron, Fortune des Contes des Grimm en France, p. 83.

6. Yann Legendre a par exemple illustré la couverture de la traduction française du roman Taxi Driver de Richard Elman, parue en 2013 aux Éditions Inculte.

7. J. et W. Grimm, Contes pour les enfants et la maison.

8. A. Defrance, «Introduction », p. 11.

9. M. Arrivé, «L'intelligence des images », §4.

10. F. Fièvre, «L'image dans l'image », p. 145.

11. Ibid., p. 146.

12. «L'œuvre de Kirby est si audacieuse qu'elle fait l'effet d'une bombe nucléaire qui nous explose au visage. Mon style consiste à combiner ce trait ancien à un outil plus contemporain ». Notre traduction. Entretien avec Scott Kirkwood (« Yann Legendre's Modern-Day Woodcuts »).

13. «Dans les temps anciens [...] vivait un roi. Ses filles étaient toutes belles, mais la plus jeune était si belle que le soleil lui-même [...]». Y. Legendre, Grimm, Contes choisis, p. 149. De nombreuses illustrations tirées du recueil sont visibles en ligne sur le site de Yann Legendre (<http://www.yannlegendre.com/project/grimms-fairy-tales/>, consulté le 27 mars 2018).

14. L. Simonson, Covergirls, p. 57. 
15. Y. Legendre, Grimm, Contes choisis, p. 98.

16. Ibid., p. 28.

17. Ch. Perrault, «Cendrillon », in Histoires, ou Contes du tems passé, p. 51 sq.

18. On peut voir cette estampe sur Gallica : <https://gallica.bnf.fr/ark:/12148/btv1b10322255v>, consulté le 12 février 2019.

19. Idem : <https://gallica.bnf.fr/ark:/12148/bpt6k9739017d/f12>, consulté le 12 février 2019. Voir encore l'illustration d'Henri Thiriet (<https://gallica.bnf.fr/ark:/12148/bpt6k9656851k/ f11.image>, consulté le 12 février 2019).

20. Ch. Perrault, «Cendrillon », dans Histoires, ou Contes du tems passé, p. 52.

21. L'expression est empruntée à A. Gunthert : "L'image conversationnelle », p. 61.

22. M. Arrivé, «L'intelligence des images ", p. 3. L'auteur de cet article parle aussi, ce qui est tout à fait pertinent dans notre cas, de la « globalisation croissante des échanges iconiques ", p. 6.

23. Y. Legendre, Grimm, Contes choisis, p. 66.

24. De nombreuses photographies de ce chat (qui est en réalité une femelle) sont visibles sur le compte Instagram dédié à l'animal (<https://www.instagram.com/realgrumpycat/?hl=fr>, consulté le 27 mars 2018).

25. M. Arrivé, «L'intelligence des images», p. 12.

26. Ibid., p. 14.

27. Y. Legendre, Grimm, Contes choisis, p. 194 et 199.

28. Entretien avec Scott Kirkwood (« Yann Legendre's Modern-Day Woodcuts »). « Dans l'histoire, rien ne dit que la jeune fille est fragile ni qu'elle a peur du loup, c'est pourquoi je n'ai pas voulu la représenter comme une fleur frêle ", dit l'illustrateur. Legendre l'a représentée comme une jeune femme déterminée dans son vêtement à capuche - et comme un personnage qui semble tout droit venu des rues de New York et prêt à affronter le mal qui se cache sous les draps de sa grandmère.

29. Y. Legendre, Grimm, Contes choisis, p. 87.

30. M. Arrivé, «L'intelligence des images », p. 3.

31. Ces illustrations sont visibles en ligne sur Gallica (<http://gallica.bnf.fr/ark:/12148/ btv1b103221759.r=dor\%C3\%A9\%20chaperon\%20rouge?rk=21459;2>) et sur le site de la BNU (<http://www.bnu.fr/images/gustave-dore-le-petit-chaperon-rouge-huile-sur-

toile-65x82cm-1862-national-gallery-victoria-melbourn?size=_original>, consulté le 27 mars 2018).

32. Entretien avec Scott Kirkwood: "Si vous voulez apprendre des choses sur l'art du XIV ${ }^{\mathrm{e}}$ au $\mathrm{XVIII}^{\mathrm{e}}$ siècle, il faut aller en France, mais si vous voulez vous former sur l'illustration contemporaine, il faut aller aux États-Unis [...]. Dans les années 1950 et 1960, la création américaine a explosé et je continue à trouver des sources d'inspiration dans des couvertures de livres réalisées par Chip Kidd, dans des romans graphiques et dans les œuvres d'art américaines, quelles qu'elles soient ».

33. Y. Legendre, Grimm, Contes choisis, p. 98.

34. Ibid., p. 148 et 178.

35. Ibid., p. 56.

36. Entretien avec Scott Kirkwood : « Les histoires des Grimm avaient vocation à être dites et non pas lues. Elles sont écrites dans un style oral et c'est justement parce qu'elles contiennent si peu de détails que je peux créer le détail et interpréter l'histoire à travers mes illustrations ».

37. Y. Legendre, Grimm, Contes choisis, p. 189.

38. Entretien avec Scott Kirkwood : "La majeure partie de mon éducation en matière d'art et d'illustration vient de mes parents. Mon père était architecte et il enseignait aussi dans une école d'art. Des artistes et des gens célèbres nous rendaient visite pendant plusieurs semaines pour travailler sur des tableaux ou des sculptures, de sorte que j'ai baigné dans la créativité ». 
39. Voir de nouveau ici A. Gunthert et son « image conversationnelle » ou encore la conclusion de l'article de M. Arrivé (« L'intelligence des images », p. 20).

\section{RÉSUMÉS}

Le graphiste français Yann Legendre parvient, dans sa sélection de vingt textes des frères Grimm parue en 2014 en France et aux États-Unis, à donner au genre du conte illustré une nouvelle identité graphique placée sous le signe d'une modernité nourrie par différentes influences telles que le pop art et les comics, mais aussi par les gravures de Gustave Doré illustrant Perrault ou par le Jugendstil. Notre contribution se propose d'interroger ce qui, dans l'œuvre de Yann Legendre, relève de l'intericonicité, de la reprise et de la recomposition, et ce qui en fait l'originalité et l'actualité du fait de la combinaison de ces différents emprunts.

The French graphic designer Yann Legendre has succeeded in giving the genre of the illustrated tale a new graphic identity in Grimm, Contes choisis, published simultaneously in the United States as Grimm's Fairy Tales (2014). The picturebook is placed under the sign of a modernity nourished by various influences such as pop art and comics, but also by Gustave Doré's engravings for Perrault's contes or the Jugendstil. My contribution examines what, in Yann Legendre's work, pertains to intericonicity, stylistic citation and recomposition, and what makes his work original and topical as a result of the innovative and bold combination of these various borrowings.

\section{AUTEUR}

\section{ANNE-SOPHIE GOMEZ}

Université Clermont Auvergne, CELIS 